\title{
Logical approach to the quantum-to-classical transition
}

Sebastian Fortin, Manuel Gadella, Federico Holik and Marcelo Losada

\section{Introduction}

The description of the classical limit of a quantum system is one of the most important issues in the foundations of quantum mechanics [1]. This problem has been formulated in different ways and explained by appealing to different interpretations [2]. The attempts to explain the classical limit go back to the correspondence principle, proposed by Niels Bohr. This principle establishes a connection between quantum observables and their classical counterparts when Planck's constant is small enough in comparison with relevant quantities of the quantum system. In particular, this happens in the limit of large quantum numbers.

Nowadays, the most important approach for describing the classical limit is based on the decoherence process [2]. The general idea of this approach is to explain the disappearance of the interference terms of quantum states appealing to the decoherence process induced by the environment. In this way, the coherence needed for most typical quantum phenomena is lost, and the classical features appear instead.

As is well known, the set of observables associated to a quantum system forms a noncommutative algebra. This differs with the classical description of physical systems, in which observables are represented by functions over a phase space, which form a commutative algebra. This difference between quantum and classical systems has a correlate in terms of the elementary properties of physical systems. The elementary properties of quantum systems (also known as Yes-No tests or elementary experiments) are represented by orthogonal projectors acting on a Hilbert space. These projectors form a non-Boolean lattice (more specifically: a complete, atomic, atomistic, orthomodular lattice, satisfying the covering law [3]). Instead, the elementary properties of a classical system are the measurable subsets of the phase space, which form a Boolean lattice.

The decoherence approach to the quantum-to-classical transition is based on the Schrödinger picture, in which states evolve over time, while observables and physical 
properties are taken to be constants. As a result, the structure of quantum properties remains the same for all times: the quantum logic associated with the system does not change [4-7]. Therefore, in this approach it is not explained how the structure of quantum properties becomes classical. However, as it was remarked in [5], a reasonable condition for the existence of a classical limit is that the lattice of elementary properties becomes Boolean, or equivalently, that the algebra of observables becomes commutative $[4,6]$.

In this chapter, we present a logical approach to the classical limit, which describes how the logical structure of the elementary properties of a quantum system becomes classical when the classical limit is reached. In order to describe the evolution of logical structure, we consider the Heisenberg picture. According to this picture, observables and physical properties evolve in time, while states remain constant. In this way, we can consider the algebra of observables and the lattice of elementary properties as a dynamical object, depending on time, or other relevant parameters, such as action, temperature, particle number or energy.

As we show below, this offers an interesting perspective for studying different physical processes. In particular, we discuss the possibility of connecting the approach of dynamical algebras developed in [4-7] with the description of the classical limit based in deformation of algebras. We also discuss the case of quantum statistical mechanics, where intermediate logics are interpreted as phase transitions.

The chapter is organized as follows. In section II, We review the problem of the classical limit, as it was traditionally considered in the literature. In section III, we briefly summarize the logical structure of the elementary properties of physical systems, and we discuss the main difference between both logical structures. In Section IV, we introduce the logical approach to the classical limit, and we illustrate this approach with four different examples. Finally, in Section V we draw our conclusions.

\section{Different approaches to the classical limit}

One of the first explanations was proposed by Niels Bohr appealing to the correspondence principle. This principle establishes a connection between quantum observables and their classical counterparts by asserting that, if the ratio between the action of the system and the Planck's constant is large enough, the classical limit should be recovered [2]. This implies that the quantum-to-classical transition should be attained in the limit of large quantum 
numbers, such as large orbits, large energies, or a large number of particles. This approach is still important nowadays, in particular for studying quantum phenomena in the semiclassical level. A result that goes in line with the correspondence principle is the Ehrenfest theorem.

Paul Dirac proposed another explanation of the classical limit, appealing to the destructive interference among all possible paths [8]. In this way, he showed that the classical action path has the dominant contribution. This idea was subsequently elaborated by Richard Feynman in his thesis [9], opening the door to the celebrated path integral formulation of quantum mechanics.

All these approaches presented problems, which where extensively discussed in the literature. In particular, it is important to remark that Bohr himself did not considered the classical limit as an explanation of the emergence of classical reality. Quite on the contrary, Bohr believed that the classical realm exists independently of quantum theory and that cannot be derived from it. As it is well known, the discussion about the classical limit is subtle and problematic, and there is no real agreement on a solution [2].

Nowadays, the most important approach for describing the classical limit is based on the environment induced decoherence. In this approach, it is considered that the quantum-toclassical transition is the result of the loss of coherence of the system due to the interaction with its environment [2]. Many physicists considered this proposal as the correct explanation of the classical limit (and also of the measurement process). However, some objection were raised, because the decoherence process would not be explaining how the logical structure of the elementary properties becomes a classical logic.

Another important approach to the study of the classical limit is based in algebras deformation [10]. In this formalism, quantum commutators (or equivalently, Moyal brackets) reduce to Poisson brackets, deforming the algebra involved.

In what follows, we present an alternative approach to describe the classical limit. This is a logical approach, based on the evolution of the quantum observables, and it allows to describe the quantum-to-classical transition of the logical structure of the quantum systems. In the next section, we review some basic features about the lattice of the elementary properties of classical and quantum systems, which are relevant to our logical approach of the classical limit. 


\section{Logical structure of quantum mechanics}

In classical and quantum mechanics, the physical properties of a system are endowed with a lattice structure. These structures are different in the classical and quantum case, and they determine the logical structure of the whole physical system. In classical mechanics, physical systems are represented by the phase space and their properties are represented by subsets of the phase space [3]. In quantum mechanics, the physical systems are represented by Hilbert spaces and the properties are represented by closed vector subspaces or by their corresponding orthogonal projectors [11] (for a recent discussions about the logic approach to quantum mechanics see [12-16], and for applications to quantum histories see [17-23]). In both cases the set of all properties of a system has an orthocomplemented lattice structure. This implies that there is an order relation $(\leq)$ such that for all pair of properties exists the infimum $(\Lambda)$ and the supremum $(\mathrm{V})$, and all property $p$ has a complement $p^{\perp}$ with adequate properties. All orthocomplemented lattices satisfy certain inequalities, called distributive inequalities [3]:

$$
\begin{aligned}
& a \wedge(b \vee c) \geq(a \wedge b) \vee(a \wedge c), \\
& a \vee(b \wedge c) \geq(a \vee b) \wedge(a \vee c)
\end{aligned}
$$

When the equalities hold, the lattice is distributive. An orthocomplemented and distributive lattice is called a Boolean lattice. The distributive property is an essential feature which differentiates classical and quantum lattices of properties.

In the classical case, the properties of the system are represented by the subsets of its phase space. The partial order relation is given by the inclusion $(\subseteq)$ of sets. The infimum and the supremum are the intersection $(\cap)$ and the union $(U)$ of sets, respectively; and the complement of a property $\mathrm{p}$ is the complement of sets $p^{\mathrm{c}}$. The set of classical properties is not only an orthocomplemented lattice, but also a distributive one, i.e., classical properties satisfy the distributive equalities. Therefore, the logical structure of a classical system is Boolean. This structure is usually called classical logic.

The quantum case is very different. The properties are represented by closed vector subspaces (or by their corresponding orthogonal projectors) [11]. Thus, the logical structure of quantum systems is given by the algebraic structure of closed vector subspaces. The set of all quantum properties is also an orthocomplemented lattice, and, as in the classical case, the partial order relation is given by the inclusion of subspaces and the infimum is given by the intersection of subspaces. However, the supremum and the complement of properties are 
different from the classical ones. The supremum is given by the sum of subspaces and the complement of a property is its orthogonal subspace. The resulting lattice is non-distributive [3], and therefore, it is not Boolean. This structure is called quantum logic [24]

The distributive inequalities are the main difference between classical and quantum logic. In the classic lattice, all properties satisfy the distributive equalities, but in the quantum lattice, only distributive inequalities hold in general. However, for some subsets of quantum properties the equalities hold. When a subset of properties satisfies the distributive equalities, they are called compatible properties. It can be proved that a sufficient and necessary condition for a set of properties to be compatible is that the projectors associated with the properties commute. Moreover, it can be shown that properties associated with different observables are compatible if the observables commute. If, on the contrary, two observables do not commute, some of the properties associated with them are not compatible. Therefore, by extension, commuting observables are called compatible observables.

The differences between classical and quantum logic are of fundamental importance for the classical limit problem. If a quantum system undergoes a physical process such that its behavior becomes classic, then its logical structure of properties should undergo a transition from quantum logic to classical logic, i.e. its lattice structure should become distributive. However, the description of the classical limit of a quantum system usually focuses on the state of the system. The mathematical description of this process does not explain how the logical structure changes on time. Therefore, in these approaches it is not possible to describe how the structure of quantum properties becomes classical. In order to give an adequate approach to the classical limit, we need a description in which observables and physical properties evolve over time, changing the logical structure of the system.

\section{Logical quantum-to-classical transition}

A complete description of the quantum-to-classical transition should explain how the logical structure of the system changes from a quantum logic to a classical logic. In order to describe adequately this transition, we consider a quantum system with a general time evolution, and a time-dependent set of relevant observables, $\mathcal{O}(t)=\left\{\hat{O}_{i}(t)\right\}_{i \in I}$ (with I a set of indexes).

Each set of relevant observables $\mathcal{O}(t)$ generates an algebra of observables $\mathcal{V}(t)$, and each algebra has associated an orthocomplemented lattice of properties $\mathcal{L}_{\mathcal{V}(t)}$. We assume that in the initial set of observables $\mathcal{O}(0)$ there are some incompatible observables, and therefore 
its corresponding algebra of observables $\mathcal{V}(0)$ is non-commutative and the associated lattice of properties $\mathcal{L}_{\mathcal{V}(0)}$ is non-distributive.

For a quantum system with a decoherence time $t_{D}$, the quantum-to-classical transition is characterized by a process that transforms non-commutative observables into commutative ones,

$$
\left[\hat{O}_{i}(0), \hat{O}_{j}(0)\right] \neq 0 \rightarrow\left[\hat{O}_{i}\left(t_{D}\right), \hat{O}_{j}\left(t_{D}\right)\right]=0, \forall i, j
$$

After time $t_{D}$, the algebra of observables $\mathcal{V}(t)$ becomes commutative, and the corresponding orthomodular lattice $\mathcal{L}_{\mathcal{V}(t)}$ becomes non-distributive. The logical classical limit is expressed by the fact that, while $\mathcal{L}_{\mathcal{V}(0)}$ is a non-distributive lattice, $\mathcal{L}_{\mathcal{V}\left(t_{D}\right)}$ is a Boolean one. In this way, we obtain an adequate description of the logical evolution of a quantum system.

In what follows, we discuss the dynamics of the quantum algebra of observables and the logic structure of properties in some physical models.

\section{A. Quantum operations}

A quantum operation is a linear and completely positive map from the set of density operators into itself [25]. For each time $t$, we consider a quantum operation $\varepsilon_{t}$, which maps the initial state of the system $\hat{\rho}_{0}$ to the state at time $t$, i.e.,

$$
\varepsilon_{t}\left(\hat{\rho}_{0}\right)=\hat{\rho}
$$

If we use the sum representation, we can express the quantum operation $\varepsilon_{t}$ as follows [25],

$$
\varepsilon_{t}\left(\hat{\rho}_{0}\right)=\sum_{\mu} \hat{E}_{\mu}(t) \hat{\rho}_{0} \hat{E}_{\mu}^{\dagger}(t),
$$

where $\hat{E}_{\mu}(t)$ are the Kraus operators of $\mathcal{E}_{t}$.

We define the Heisenberg representation of $\varepsilon_{t}$ as the operator $\tilde{\varepsilon}_{t}$ that evolves the observables from an initial time up to time $t$, i.e., $\tilde{\varepsilon}_{t}(\hat{O})=\hat{O}(t)$. The operator $\tilde{\varepsilon}_{t}$ must preserve the mean values of the observables for all times,

$$
\operatorname{Tr}(\hat{\rho}(t) \hat{O})=\operatorname{Tr}\left(\sum_{\mu} \hat{E}_{\mu}(t) \hat{\rho}_{0} \hat{E}_{\mu}^{\dagger}(t) \hat{O}\right)=
$$




$$
=\operatorname{Tr}\left(\hat{\rho} \sum_{\mu} \hat{E}_{\mu}^{\dagger}(t) \hat{O} \hat{E}_{\mu}(t)\right)=\operatorname{Tr}\left(\hat{\rho}_{0} \hat{O}(t)\right)
$$

Hence, we can express the operator $\tilde{\varepsilon}_{t}$ is the following

$$
\tilde{\varepsilon}_{t}(\hat{O})=\hat{O}(t) \sum_{\mu} \hat{E}_{\mu}^{\dagger}(t) \hat{O} \hat{E}_{\mu}(t)
$$

Since $\hat{O}$ is self-adjoint, then $\tilde{\varepsilon}_{t}(\hat{O})$ is also a self-adjoint operator. Therefore, $\tilde{\varepsilon}_{t}$ maps observables to observables.

Once we have defined the temporal evolution of quantum operations, we can describe the logical classical limit of a quantum system as it was explained before. We illustrate the logical approach with a simple example: the amplitude damping channel [25].

The amplitude damping channel is useful for describing the energy dissipation due to the environment effects. It is relevant for quantum information processing, because it is an adequate model for quantum noise. In particular, this model can be applied to the decay of an excited state of a two-level atom due to spontaneous emission of photons. If the atom is in the ground state, no photon is emitted, and the atom continues in the same state. But, if the atom is in the excited state, after an interval of time $\tau$, there is a probability $\mathrm{p}$ that the state has decayed to the ground state and a photon has been emitted [25].

The quantum operation of the amplitude damping channel can be expressed as follows

$$
\varepsilon_{t}\left(\hat{\rho}_{0}\right)=\widehat{E}_{0} \hat{\rho}_{0} \hat{E}_{0}^{\dagger}+\widehat{E}_{1} \hat{\rho}_{0} \hat{E}_{1}^{\dagger}
$$

where the Kraus operators are

$$
\widehat{E}_{0}=\left(\begin{array}{cc}
1 & 0 \\
0 & \sqrt{(1-P)}
\end{array}\right), \hat{E}_{1}=\left(\begin{array}{ll}
0 & p \\
0 & 0
\end{array}\right)
$$

The associated quantum map $\tilde{\varepsilon}_{t}$ acting on the space of observables is given by $\tilde{\varepsilon}_{t}(\hat{O})=$ $\hat{E}_{0}^{\dagger} \hat{\rho}_{0} \widehat{E}_{0}+\hat{E}_{1}^{\dagger} \hat{\rho}_{0} \hat{E}_{1}$. In matrix form, we have

$$
\tilde{\varepsilon}_{t}(\hat{O})=\left(\begin{array}{cc}
O_{00} & \sqrt{(1-p)^{n}} O_{01} \\
\sqrt{1-p} O_{10} & p O_{00}+(1-p) O_{11}
\end{array}\right)
$$

Applying the amplitude damping channel $\mathrm{n}$ times, we obtain the map $\widetilde{\varepsilon}_{n \tau}(\hat{O})$, which has the form 


$$
\widetilde{\varepsilon}_{n \tau}(\hat{O})=\left(\begin{array}{cc}
O_{00} & \sqrt{(1-p)^{n}} O_{01} \\
\sqrt{(1-p)^{n}} O_{10} & (1-p)^{n} O_{11}+O_{00}\left[1-(1-p)^{n}\right]
\end{array}\right) .
$$

If $\mathrm{t} \rightarrow \infty$, all observables become proportional to the identity. This implies that the algebra of observables becomes trivially commutative, and its corresponding lattice of properties becomes a classical logic.

\section{B. Rigged Hilbert space}

One of the most investigated fields in quantum foundations is the quantization problem, which consists in obtaining quantum observables from their classical counterpart. Much less considered has been the problem of de-quantization: the transition form quantum to classical observables. It was shown in [26] that de-quantization may require two steps, one is a type of decoherence and the other is the notion of macroscopicity, which is implemented by the limit $\hbar \rightarrow 0$. Along the present section, we intend to give a brief account of another notion of dequantization in presence of unstable quantum systems or resonances.

In a previous paper [6], we argued that an essential characteristic of the quantum-toclassical transition should be the transition from a non-commutative algebra of observables to ia commutative one, when $t \rightarrow \infty$. This can be rigorously formulated for quantum unstable systems, provided we considered the linear space spanned by the resonance state vectors, also called Gamow vectors.

Resonances are usually characterized as poles of some analytic continuations of a reduced resolvent or a scattering matrix. Both formulations are not always equivalent: one may construct models for which the poles in one of these two formulations are not poles in the other. In the energy representation, these poles appear in complex conjugate pairs and have the form $E_{R} \pm i \Gamma / 2$, where $E_{R}$ is the resonance energy and $\Gamma$ is related with the inverse of the mean life [27]. Notice that $\Gamma$ must be always positive.

From the observational point of view, single resonances show an exponential decay, provided that the time intervals are not too short and not very large either [28]. However, these deviations are very difficult to be observed. Therefore, most experiments with resonances show exponential decays for practically all values of time [29, 30].

Now, pure stable states have a mathematical representation in terms of vector states. The difference between a stable state and a resonance one is just that the value of the parameter $\Gamma$ is equal to zero for stable states. Then, one is tempted to introduce a definition of 
resonance states in such a way that, if the resonance poles are $E_{R} \pm i \Gamma / 2$, we have either $H\left|\psi^{D}\right\rangle=\left(E_{R}-i \Gamma / 2\right)\left|\psi^{D}\right\rangle$ or $H\left|\psi^{G}\right\rangle=\left(E_{R}+i \Gamma / 2\right)\left|\psi^{G}\right\rangle[31]$. Here $H=H_{0}+V$ is the total Hamiltonian which produces the resonance phenomenon. Note that, in the first case, formal time evolution gives $e^{-i t H}\left|\psi^{D}\right\rangle=e^{-i E_{R} t} e^{-t \Gamma / 2}\left|\psi^{D}\right\rangle$, which is an exponential decay for $t \rightarrow$ $\infty$. On the other hand, a similar formal time evolution gives $e^{-i t H}\left|\psi^{G}\right\rangle=e^{-i E_{R} t} e^{t \Gamma / 2}\left|\psi^{D}\right\rangle$, which decays exponentially as $t \rightarrow-\infty$. Vector states $\left|\psi^{D}\right\rangle$ and $\left|\psi^{G}\right\rangle$ as known as the decaying and growing Gamow vectors, respectively.

As a matter of fact, both vectors $\left|\psi^{D}\right\rangle$ and $\left|\psi^{G}\right\rangle$ (where the D stands for decay and the $\mathrm{G}$ stands for growing) are equally suitable for a vector state for the considered resonance. Nevertheless, the choice $\left|\psi^{D}\right\rangle$ seems more natural as the time flows in the positive direction. The point is that both are time reversal of each other and represent the same physical phenomenon. Note that, both vector states describe the part of the resonance that behaves exponentially with time. Deviations add a background term [32], but here we can consider as negligible.

However, the previous considerations have an important mathematical flaw: Gamow vectors are eigenvectors of the total Hamiltonain $H$ with complex eigenvalues. This is not compatible with the assumption that $H$ is self adjoint. However, this property is essential if we want that the Gamow vectors have an exponential behavior with time. There are two possible remedies for this problem:

1. Non-Hermitian Hamiltonian

This is the approach known as dilation analytic potentials [33]. This gives normalizable Gamow vectors belonging to the Hilbert space on which the total Hamiltonian $H$ is defined as a self-adjoint operator. However, these Gamow vectors depend on a nonphysical parameter, precisely the parameter that provides the dilation, which is arbitrary at some extent [34]. There are other possibilities for using non-Hermitian Hamiltonians (see for instance [35]).

\section{Rigged Hilbert space}

The second possibility is the extension of the Hilbert space to a rigged Hilbert space (RHS). A RHS is a tern of three spaces $\Phi \subset \mathcal{H} \subset \Phi^{\times}$, with the following properties: i.) 
$\mathcal{H}$ is an infinite dimensional separable Hilbert space ${ }^{1}$; ii) $\Phi$ is a dense subspace ${ }^{2}$ with a topology such that the $\Phi$ has less convergent sequences than what it would have with the topology inherited from $\mathcal{H}$; iii) $\Phi^{\times}$is the vector space of all continuous linear mappings from $\Phi$ to the space $\mathbb{C}$ of complex numbers. RHS also serves for a rigorous presentation of the Dirac formulation of quantum mechanics [36-41], and it has some other applications concerning group representations and special functions [42-44].

Then, if $\mathcal{H}$ is the Hilbert space where the total Hamiltonian $H=H_{0}+V$ acts on, we may construct two RHS, $\Phi_{ \pm} \subset \mathcal{H} \subset \Phi^{\times}$, with the property that $\left|\psi_{j}^{D}\right\rangle \in \Phi_{+}^{\times}$and $\left|\psi_{j}^{G}\right\rangle \in \Phi_{-}^{\times}$, where the index $j$ stands for the number of resonances in the system with resonance complex energies $E_{R_{j}} \pm i \Gamma_{j} / 2$. Decaying and growing Gamow vectors have the desired time behavior, a fact that can be rigorously proven $[32,45]$

A non-relativistic quantum system may have infinitely many resonances. This means that only a finite number of resonances may be considered. We recall that resonances are determined by the poles of a complex analytic function which are always isolated points in the complex plane. For large values of $E_{R}$, the energies go to the relativistic regime, so that we have to discard this possibility. But then, resonances with large imaginary part are not observable, since their mean lifetimes are extremely small. This means that only a finite number of resonances may be considered within the non-relativistic regime for a given unstable quantum system.

In addition, if we focus our attention in the resonance behavior only, we may consider the space spanned by the Gamow vectors. For decaying (growing) Gamow vectors, this is a finite dimensional subspace of $\Phi_{+}^{\times}\left(\Phi_{-}^{\times}\right)$. Let us assume that our system has $N$ resonances with $z_{j}:=E_{R_{j}}+i \Gamma_{j} / 2$ and $z_{j}^{*}$ being its complex conjugate. We may consider the $2 N$ dimensional space $\mathcal{H}^{G}$ spanned by all Gamow vectors

$$
\left\{\left|\psi_{1}^{D}\right\rangle,\left|\psi_{2}^{D}\right\rangle,\left|\psi_{1}^{G}\right\rangle,\left|\psi_{2}^{G}\right\rangle, \ldots,\left|\psi_{N}^{D}\right\rangle,\left|\psi_{N}^{G}\right\rangle\right\}
$$

We define on $\mathcal{H}^{G}$ a pseudometrics that on the vectors of the basis (2) is

\footnotetext{
${ }^{1}$ A Hilbert space is separable if any orthonormal basis is countable.

${ }^{2}$ A subspace of $\mathcal{H}$ is dense if any neighborhood of any vector in $\mathcal{H}$ contains vectors in $\Phi$.
} 


$$
\left(\psi_{i}^{D} \mid \psi_{j}^{D}\right)=\left(\psi_{i}^{G} \mid \psi_{j}^{D}\right)=0, \quad\left(\psi_{i}^{D} \mid \psi_{j}^{G}\right)=\left(\psi_{i}^{G} \mid \psi_{j}^{D}\right)=\delta_{i j},
$$

where $\delta_{i j}$ is the Kronecker delta. We extend this pseudometrics to the whole of $\mathcal{H}^{G}$ by linearity.

We may write the restriction of the total Hamiltonian $H$ to $\mathcal{H}^{G}$ as [46]

$$
\left.H=\sum_{j=1}^{N} z_{j} \mid \psi_{i}^{D}\right)\left(\psi_{j}^{G}\left|+\sum_{j=1}^{N} z_{j}^{*}\right| \psi_{i}^{G}\right)\left(\psi_{j}^{D} \mid\right.
$$

Note that $H$ in (4) is formally Hermitian. Using the pseudometrics (3), we find that

$$
\left.H^{n}=\sum_{j=1}^{N} z_{j}^{n} \mid \psi_{i}^{D}\right)\left(\psi_{j}^{G}\left|+\sum_{j=1}^{N}\left(z_{j}^{*}\right)^{n}\right| \psi_{i}^{G}\right)\left(\psi_{j}^{D} \mid\right.
$$

This suggests a possible choice for the time evolution operator on $\mathcal{H}^{G}$ as

$$
U(t):=e^{-i t H}=\sum_{j=1}^{N}\left\{e^{-i t z j} \mid \psi_{i}^{D}\right)\left(\psi_{j}^{G}\left|+e^{-i t z_{j}^{*}}\right| \psi_{i}^{G}\right)\left(\psi_{j}^{D} \mid\right\}
$$

The identity $I$ on $\mathcal{H}^{G}$ in this representation is given by

$$
I:=\sum_{j=1}^{N}\left\{\mid \psi_{j}^{D}\right)\left(\psi_{j}^{G}|+| \psi_{j}^{G}\right)\left(\psi_{j}^{D} \mid\right\}
$$

Using the pseudometrics, we obtain that $\left.\left.I \mid \psi_{j}^{D}\right)=\mid \psi_{j}^{D}\right)$ and $\left.I\left|\psi_{j}^{G}\right\rangle=\mid \psi_{j}^{G}\right), j=$ $1, \ldots, N$, so that this is indeed the identity. With this identity, one possible choice of the inverse of $U(t)$ is

$$
U^{-1}(t)=U(-t)=\sum_{j=1}^{N}\left\{e^{i t z_{j}} \mid \psi_{i}^{D}\right)\left(\psi_{j}^{G}\left|+e^{i t z_{j}^{*}}\right| \psi_{i}^{G}\right)\left(\psi_{j}^{D} \mid\right\}
$$

Then, we obtain $U(t) U^{-1}(t)=I$ as we would expect.

The time evolution for any observable $O=O(0)$ on $\mathcal{H}^{G}$ should be defined as

$$
O(t):=U(-t) O U(t)
$$

This $O(t)$ is well defined for al values of time $t$. However, with definitions (6) and (8), $O(t)$ diverges as $t \rightarrow \pm \infty$. This result is not satisfactory. This is the reason why we have chosen instead, as evolution operator on $\mathcal{H}^{G}$ : 


$$
U(t):=\sum_{j=1}^{N}\left\{e^{-i t z_{j}} \mid \psi_{i}^{D}\right)\left(\psi_{j}^{G}\left|+e^{i t z_{j}^{*}}\right| \psi_{i}^{G}\right)\left(\psi_{j}^{D} \mid\right\}
$$

which is Hermitian. In this case, we have that $U(t) U^{\dagger}(t)=e^{-t \Gamma} I$. Nevertheless, we should keep the definition of $O$ at time $t$ as $O(t):=U^{\dagger}(t) O U(t)$. In this case, we have the following relationship for the commutator of two observables at time $t$ :

$$
\left[O_{1}(t), O_{2}(t)\right]=\sum_{j=1}^{N} e^{-2 t \Gamma_{j}}\left\{\alpha_{j}(t) \mid \psi_{j}^{D}\right)\left(\psi_{j}^{G}\left|+\beta_{j}(t)\right| \psi_{j}^{G}\right)\left(\psi_{j}^{D} \mid\right\}
$$

where $\alpha_{j}(t)$ and $\beta_{j}(t), i=1,2, \ldots, N$, are constants for which the dependence on $\mathrm{t}$ is just a

phase of the form $e^{ \pm 2 i t E_{R_{j}}}$. Since all $\Gamma_{j}>0$, one concludes that, in the limit $t \rightarrow \infty$, the commutator (11) vanishes.

In conclusion, for quantum decaying systems and with a correct choice of the form of our operators, commutators vanish for long values of time.

\section{Decoherence and irreversible process}

Many attempts have been made to recover the laws of classic mechanics through some classical limit. The more relevant approaches include the quantum decoherence process, which is responsible for the disappearance of the interference terms of quantum states, inadmissible for a classical description. In addition, decoherence provides a rule for choosing the candidates for classic states.

As it is indicated in [47], three periods can be identified in the development of the general program of decoherence [48]. In the first period, it was studied the arrival to the equilibrium of irreversible systems. During this period, authors such as van Kampen, van Hove, Daneri, et al. developed a formalism that was not successful for explaining the decoherence phenomenon, but it established the basis for its future development. The main problem of this period was that too long decoherence times were found, in comparison with the experimental ones.

In the second period, the decoherence in open systems was studied. The main characters of this period were Zeh and Zurek [49-52]. The decoherence process is described as interaction process between an open quantum system and its environment. This process, called Environment-Induced Decoherence (EID), determines a privileged basis (usually called 
pointer basis or moving decoherence basis) which defines the observables that acquires classic features. Nowadays, this is the orthodox position of the subject [7]. The decoherence times in this period were much smaller, solving the problem of the first period.

In the third period, the study of the arrival to equilibrium of closed systems was studied (G. Ford and R. O'Connel [53], G. Casati, B. Chirikov and T. Prosen [54-56], R. Gambini, J. Pulin and R. A. Porto [57-59], and M. Frasca [60]). Within this period, a new approach of the decoherence was presented by Castagnino et al. According to this approach, the decoherence process can occur in closed systems, and it depends on the choice of some observables with some particular physical relevance (for example, the van Hove observables). This process, called Self-Induced Decoherence (SID), also determines which is the privileged basis, called final decoherence basis, that defines which observables acquire classic features.

In some works $[47,61,62]$, the common characteristics of the different approaches to decoherence were summarized, and a general framework for decoherence was proposed. According to the general framework, decoherence is just a particular case of the general problem of irreversibility in quantum mechanics. Since the quantum state follows an unitary evolution, it cannot reach a final equilibrium state when time goes to infinity. Therefore, another element must be considered in such a way that a non-unitary evolution is obtained. The way to introduce this non-unitary evolution has to include the splitting of the whole space of observables $\mathcal{O}$ into a relevant subspace $\mathcal{O}_{R} \subset \mathcal{O}$ and an irrelevant subspace. Once the essential role played by the selection of the relevant observables is clearly understood, the phenomenon of decoherence can be explained in four general steps (we reproduce the four steps from paper [62]):

\section{- First step:}

The space of relevant observables $\mathcal{O}_{R}$ is defined. For example, in the EID approach the relevant observables are $O_{R}=O_{S} \otimes I_{E}$, where $O_{S}$ is an arbitrary observable of the system $S$ and $I_{E}$ is the unit operator of the environment $E$. SID relevant observables were defined in [62].

\section{- Second step:}

The expectation value $\left\langle O_{R}\right\rangle_{\rho(t)}$, for any $O_{R} \in \mathcal{O}_{R}$, is obtained. This step can be formulated in two different but equivalent ways: 
i. $\left\langle O_{R}\right\rangle_{\rho(t)}$, is obtained as the expectation value of $O_{R}$ in the unitarily evolving state $\rho(t)$ (this step is typical of SID) and its evolution is studied.

ii. A coarse-grained state $\rho_{R}(t)$ is defined as

$$
\left\langle O_{R}\right\rangle_{\rho(t)}=\left\langle O_{R}\right\rangle_{\rho_{R}(t)}
$$

for any $O_{R} \in \mathcal{O}$, and its non-unitary evolution, governed by a master equation, is obtained (this step is typical of EID).

\section{- Third step:}

It is proved that $\left\langle O_{R}\right\rangle_{\rho(t)}=\left\langle O_{R}\right\rangle_{\rho_{R}(t)}$ reaches a final equilibrium value $\left\langle O_{R}\right\rangle_{\rho *}$ :

$$
\lim _{t \rightarrow \infty}\left\langle O_{R}\right\rangle_{\rho(t)}=\lim _{t \rightarrow \infty}\left\langle O_{R}\right\rangle_{\rho_{R}(t)}=\left\langle O_{R}\right\rangle_{\rho *}, \quad \forall O_{R} \in \mathcal{O}_{R}
$$

This also means that the coarse-grained state $\rho_{R}(t)$ evolves, with a non-unitary evolution, towards a final equilibrium state:

$$
\lim _{t \rightarrow \infty}\left\langle O_{R}\right\rangle_{\rho_{R}(t)}=\left\langle O_{R}\right\rangle_{\rho *}, \quad \forall O_{R} \in \mathcal{O}_{R}
$$

\section{- Fourth step:}

The moving preferred basis $\{\widehat{\mid J(t)}\rangle\}$ is defined. This basis is the eigenbasis of a state $\rho_{P}(t)$ such that

$$
\lim _{t \rightarrow \infty}\left\langle O_{R}\right\rangle_{\left(\rho_{R}(t)-\rho_{P}(t)\right)}=0, \quad \forall O_{R} \in \mathcal{O}_{R}
$$

The characteristic time for this limit is the decoherence time $t_{D}$.

All the approaches to decoherence have one thing in common, they all need to introduce a non-unitary evolution. From a general point of view, it is possible to approximate the evolution of the system through an effective non-Hermitian Hamiltonian $H_{\text {eff }}$. It can be proved that the evolution of the mean value is given by [63]

$$
\left\langle O_{R}\right\rangle_{\rho(t)} \cong\left\langle O_{R}\right\rangle_{\rho *}+\sum_{i} C_{i} e^{-\gamma_{i} t}
$$

where $\gamma_{i}^{-1}$ are the characteristic times of the system, which are associated with the complex $\mathrm{i}$ eigenvalues of the effective Hamiltonian. Then, it is easy to see that the commutator between two relevant observables is [5]: 


$$
\left\langle\left[O_{R}, O_{R}^{\prime}\right]\right\rangle_{\rho(t)} \rightarrow 0
$$

This means that, when $t \rightarrow \infty$, the expectation value of the commutator between $O_{R}$ and $O^{\prime}{ }_{R}$ becomes zero. Therefore, the Heisenberg uncertainty relation becomes undetectable from the experimental viewpoint.

\section{Quantum statistics and the classical limit}

In this section we show that the dynamics of logics can be related to other parameters, different from time. In quantum statistics, the mean number of particles occupying a quantum state is given by the formula

$$
\bar{n}_{s}=\frac{1}{\exp \left(\alpha+\beta \epsilon_{s}\right) \pm 1}=N
$$

in which the "+" sign corresponds to Fermi-Dirac statistics and the "-" to Bose-Einsten. The parameter $\alpha$ is related to particle number according to the condition

$$
\sum_{s} \bar{n}_{s}=\sum_{s} \frac{1}{\exp \left(\alpha+\beta \epsilon_{s}\right) \pm 1}=N
$$

being $N$, the total particle number. The partition function reads

$$
\ln (Z)=\alpha N \pm \sum_{s} \ln \left(1 \pm \exp \left(-\alpha-\beta \epsilon_{S}\right)\right)
$$

When the concentration of the gas is made sufficiently low, quantum effects should be important. This limit corresponds to small $N$. Equivalently, we should have $\bar{n}_{s} \ll 1$ (or $\left.\exp \left(\alpha+\beta \epsilon_{s}\right) \gg 1\right)$.

If we now assume that the particle number is fixed, and increase the temperature (this is equivalent to $\beta \rightarrow 0$ ), we obtain that the most important terms are those satisfying $\beta \epsilon_{s} \ll$

$\alpha$. Under these conditions, we obtain that $\exp \left(\alpha+\beta \epsilon_{s}\right) \gg 1$. Or equivalently, that $\bar{n}_{s} \ll$ 1. This is the condition for the classical limit. In other words, the condition under which quantum effects are negligible. In this limit, and for both cases, Fermi-Dirac and BoseEinstein, we obtain

$$
\bar{n}_{s}=\exp \left(-\alpha-\beta \epsilon_{s}\right)
$$

The above constraint reduces to 


$$
\sum_{s} \exp \left(-\alpha-\beta \epsilon_{s}\right)=N
$$

then, we can express

$$
\bar{n}_{s}=N \frac{\exp \left(-\alpha-\beta \epsilon_{s}\right)}{\sum_{s} \exp \left(\beta \epsilon_{s}\right)} .
$$

Thus, at sufficiently low density or high temperature, we obtain the Maxwell-Boltzmann distribution, which is a signature of classicality. But the fact that we can attain the classical limit by adjusting the temperature, suggests that, time is no the only parameter that allows us to observe a dynamical logics phenomenon. The algebraic aspects of this transition will be discussed in a future work. But we can advance some points here.

First, some interpretations of quantum mechanics suggest that, when the classical limit is obtained, irreversible process should be observed. Under this perspective, This can be related to the mathematical formalism of Gamow vectors.

Our second remark is that the dynamics of logics approach can be useful to interpret the quantization deformation formalism under a new light. Indeed, some authors [10] have proposed to study the classical limit and the quantization of a given theory by appealing to the formalism of deformation quantization. In this approach one starts with a classical (commutative) algebra of observables $\mathcal{A}_{0}$, endowed wit a pointwise product $\cdot$ and a Poisson bracket $\{$,$\} . Then, a family of algebras \mathcal{A}_{h}$ is introduced, indexed with a parameter $h \geq 0$. The parameter $h$ is intended to represent a dimensionless combination of some characteristic parameters associated to the system, and the Planck's constant. An associative product $\star_{h}$ is introduced in the indexed algebras, and it is required that (see [10] for details):

$$
\lim _{h \rightarrow 0} \frac{i}{h}[f, g]_{-}^{h}=\{f, g\}
$$

and

$$
\frac{1}{2} \lim _{h \rightarrow 0}[f, g]_{+}^{h}=f \cdot g
$$

The examples shown in this section suggest that the parameters involved in the classical limiting process could be time, temperature, particle number, etc. Thus our dynamical logics approach could be connected in a natural way to that based in the deformation of algebras. We will discuss this possibility elsewhere. 


\section{Conclusions}

In this chapter, we have presented a logical approach for the description of the quantum- to-classical transition of physical systems. This approach consists in describing the system as a collection of observables which evolve over time, according to the Heisenberg picture but with a non-unitary evolution.

In turn, the algebra of observables determines a lattice of elementary physical properties with a logical structure. In the classical case, the properties have classical logic structure, and in the quantum case, they have a quantum logic structure. The time evolution of the algebra induces a time evolution of the lattice of properties. Therefore, in this approach, the classical limit is attained when the final structure of properties becomes a classical logic, or equivalently, when the resulting algebra of observables becomes commutative.

We have shown some examples in which this logical transition occurs, among them, quantum channels, unstable physical processes and models of self-induced decoherence. We have also shown that our formulation has a natural application in quantum statistical mechanics, where the temperature parameter or the particle number can play the role of the time in reaching the classical limit. In other words, the classical limit of quantum statistical systems indicates that, time is not the only parameter that may show a transition from quantum to classical logic. Furthermore, we have connected our approach with the formalism of quantization deformation. In future works, we will develop these ideas in more detail.

\section{References}

[1] Cohen, D. (1989). An Introduction to Hilbert Space and Quantum Logic. Berlin: Springer-Verlag.

[2] Schlosshauer, M. (2007). Decoherence and the Quantum-to-Classical Transition. Berlin: Springer.

[3] Kalmbach, G. (1983). Orthomodular Lattices. San Diego: Academic Press.

[4] Losada, M., Fortin, S., Holik, F. (2018) Classical limit and quantum logic. International Journal Of Theoretical Physics 57: 465-475.

[5] Fortin, S., Vanni, L. (2014) Quantum decoherence: a logical perspective. Foundations of Physics 44: 1258-1268. 
[6] Fortin, S., Holik, F., Vanni, L. (2016) Non-Unitary Evolution of Quantum Logics. Springer Proceedings in Physics 184: 219-234.

[7] Bub, J. (1997). Interpreting the Quantum World. Cambridge: Cambridge University Press.

[8] Dirac, P. A. M. (1933). The Lagrangian in quantum mechanics. Physikalische Zeitschrift der Sowjetunion 3: 64-72.

[9] Feynman, R. P. (1942) The Principle of Least Action in Quantum Mechanics. Princeton University. Reproduced in Feynman, R. P. (2005). Brown, L. M. (ed.) Feynman's Thesis: a New Approach to Quantum Theory. World Scientific.

[10] Landsman, N. P. (1993) Deformation of algebras of observables and the classical limit of quantum mechanics. Reviews in Mathematical Physics 5: 775-806.

[11] von Neumann, J. (1932.) Mathematische Grundlagen der Quantenmechanik. Heidelberg: University Press.

[12] Domenech, G., Holik, F., Massri, C. (2010) A quantum logical and geometrical approach to the study of improper mixtures. Journal of Mathematical Physics 51: 052108.

[13] Holik, F., Massri, C., Ciancaglini, N. (2012) Convex Quantum Logic. International Journal of Theoretical Physics 51: 1600-1620.

[14] Holik, F., Massri, C., Plastino, A., Zuberman, L. (2013). On the Lattice Structure of Probability Spaces in Quantum Mechanics. International Journal of Theoretical Physics 52: 1836-1876.

[15] Holik, F., Plastino, A., Sáenz, M. (2014). A Discussion on the Origin of Quantum Probabilities. Annals of Physics 340: 293-310.

[16] Holik, F., Plastino, A. (2015) Quantum Mechanics: A New Turn in Probability Theory. Pp. 399-414, in Z. Ezziane (ed.) Contemporary Research in Quantum Systems, New York: Nova Publishers.

[17] Omnès, R. (1994) The Interpretation of Quantum Mechanics. Princeton: Princeton University Press.

[18] Griffiths, R. B. (2014). Foundations of Physics 44: 610-640.

[19] Losada, M., Vanni, L., Laura, R. (2013). Probabilities for time-dependent properties in classical and quantum mechanics. Physical Review A 87: 052128. 
[20] Losada, M., Laura, R. (2014). Quantum histories without contrary inferences. Annals of Physics 351: 418-425.

[21] Losada, M., Laura, R. (2014). Generalized contexts and consistent histories in quantum mechanics. Annals of Physics 344: 263-274.

[22] Losada, M., Vanni, L., Laura, R. (2016) The measurement process in the generalized contexts formal- ism for quantum histories. International Journal of Theoretical Physics 55: $817-824$.

[23] Losada, M. (2018) Contrary quantum histories and contrary inferences. Physica A: Statistical Mechanics and its Applications 503: 379-389.

[24] Birkhoff, G., von Neumann, J. (1936) The Logic of Quantum Mechanics. Annals of mathematics 37: 823-843.

[25] Nielsen, M., Chuang, I. (2000). Quantum Computation and Quantum Information. Cambridge: Cambridge University Press.

[26] Castagnino, M., Gadella, M. (2006) The problem of the classical limit of quantum mechanics and the role of self-induced decoherence. Foundations of Physics 36: 920952.

[27] Bohm, A. (1993). Quantum Mechanics: Foundations and Applications. Berlin and New York: Springer.

[28] Fonda, L., Ghirardi, G. C., Rimini, A. (1978) Decay theory of unstable quantum systems. Reports on Progress in Physics 41: 587-631.

[29] Fischer, M. C., Gutierrez-Medina, B., Raizen, M. G. (2001) Observation of the quantum Zeno and anti-Zeno effects in an unstable system. Physical Review Letters 87: 40402.

[30] Rothe, C., Hintschich, S. L., Monkman, A. P. (2006). Violation of the exponentialdecay law at long times. Phys. Rev. Lett., 96: 163601.

[31] Nakanishi, N., (1958) A theory of clothed unstable particles. Progress of Theoretical Physics, 19 :607-621.

[32] Bohm, A., Gadella. M., (1989) Dirac Kets, Gamow Vectors, and Gel'fand Triplets: The Rigged Hilbert Space Formulation of Quantum Mechanics. Berlin: Springer. 
[33] Balslev, E., Combes, J. M. (1971) Spectral properties of many body Schrödinger operators with dilation analytic intercations. Communications in Mathematical Physics 22: 280 .

[34] Reed, M., Simon, B. (1978) Analysis of Operators. New York: Academic.

[35] Eleuch, H., Rotter, I. (2017) Resonances in open quantum systems. Physical Review A 98: 0221117.

[36] Bohm, A. (1978) The Rigged Hilbert Space and Quantum Mechanics, Springer Lecture Notes in Physics. New York: Springer.

[37] Roberts, J. E. (1966) Rigged Hilbert spaces in quantum mechanics. Communications in Mathematical Physics 3: 98-119.

[38] Antoine, J. P. (1969) Dirac formalism and symmetry problems in quantum mechanics I: General Dirac formalism. Journal of Mathematical Physics 10: 53.

[39] Melsheimer, O. (1974) Rigged Hilbert space formalism as an extended mathematical formalism for quantum systems 1. General theory. Journal of Mathematical Physics 15: 902-916.

[40] Gadella, M., Gómez, F. (2002) A unified mathematical formalism for the Dirac formulation of quantum mechanics. Foundations of Physics 32: 815-869.

[41] Gadella, M., Gómez, F. (2003) On the mathematical basis of the Dirac formulation of quantum mechanics. Foundations of Physics 42: 2225-2254.

[42] Celeghini, E., Gadella, M., del Olmo, M. A. (2016) Applications of rigged Hilbert spaces in quantum mechanics and signal processing. Journal of Mathematical Physics 57: 072105 .

[43] Celeghini, E., Gadella, M., del Olmo, M. A. (2017) Lie algebra representations and rigged Hilbert spaces: The SO(2) case. Acta Polytechnica 57: 379-384.

[44] Celeghini, E., Gadella, M., del Olmo, M. A. (2018) Spherical harmonics and rigged Hilbert spaces. Journal of Mathematical Physics 59: 053502.

[45] Civitarese, O., Gadella, M. (2004) Physical and mathematical aspects of Gamow states. Physics Reports 396: 41-113.

[46] Losada, M., Fortin, F., Gadella, M., Holik, F. Dynamical algebras in quantum unstable systems. Preprint. 
[47] Castagnino, M., Fortin, S., Laura, R., Lombardi, O. (2008) A general theoretical framework for decoherence in open and closed systems. Classical and Quantum Gravity 25: 154002 .

[48] Omnès, R. (2005) Results and problems in decoherence theory. Brazilian Journal of Physics 35: 207-210.

[49] Zeh, H. D. (1970) On the Interpretation of Measurement in Quantum theory. Foundations of Physics 1: 69-76.

[50] Zeh, H. D. (1973) Toward a Quantum Theory of Observation. Foundations of Physics 3: $109-116$.

[51] Zurek, W. (1982) Environment-Induced Superselection Rules. Physical Review D 26: 1862-1880.

[52] Zurek, W. (1991) Decoherence and the Transition from Quantum to Classical. Physics Today 44: 36-44.

[53] Ford, G. W., O’Connel, R. F. (2001) Decoherence without dissipation. Physics Letters A 286: 87-90.

[54] Casati, G., Chirikov, B. (1995) Comment on 'Decoherence, Chaos, and the Second Law'. Physical Review Letters 75: 350.

[55] Casati, G., Chirikov, B. (1995) Physica D: Nonlinear Phenomena. Physical Review D 86: $220-237$.

[56] Casati, G., Prosen, T. (2005) Quantum chaos and the double-slit experiment. Physical Review A 72: 032111.

[57] Gambini, R., Pulin, J. (2007) Relational Physics with Real Rods and Clocks and the Measurement Problem of Quantum Mechanics. Foundations of Physics 37: 10741092.

[58] Gambini, R., Porto, R. A., Pulin, J. (2007) Fundamental decoherence from quantum gravity: a pedagogical review. General Relativity and Gravitation 39: 11431156.

[59] Gambini, R., Pulin, J. (2010) Modern space-time and undecidability. In V. Petkov (ed.), Minkowski Spacetime: A Hundred Years Later. Fundamental Theories of Physics 165, Heidelberg: Springer.

[60] Frasca, M. (2003) General theorems on decoherence in the thermodynamic limit. Physics Letters A 308: 135-139. 
[61] Castagnino, M., Lombardi, O. (2004) "elf-induced decoherence: A new approach. Studies in History and Philosophy of Modern Physics 35: 73-107.

[62] Castagnino, M., Fortin, S. (2013) Formal Features of a General Theoretical Framework for Decoherence in Open and Closed Systems. International Journal of Theoretical Physics 52: 13791398.

[63] Castagnino, M., Fortin, S. (2012) Non-Hermitian Hamiltonians in Decoherence and Equilibrium Theory. Journal of Physics A 45: 444009. 